\begin{tabular}{|c|l|}
\hline Title & Three body decay of linear-chain states in C14 \\
\hline Author(s) & Baba, T.; Kimura, M. \\
\hline Citation & $\begin{array}{l}\text { Physical Review C, 95(6), 064318 } \\
\text { https://doi.org/10.1103/PhysRevC.95.064318 }\end{array}$ \\
\hline Issue Date & 2017-06-22 \\
\hline Doc URL & http://hdl.handle.net/2115/66304 \\
\hline Rights & @2017 A merican Physical Society \\
\hline Type & article \\
\hline File Information & PhysRevC95 064318.pdf \\
\hline
\end{tabular}

Instructions for use 


\title{
Three-body decay of linear-chain states in ${ }^{14} \mathrm{C}$
}

\author{
T. Baba $^{1}$ and M. Kimura ${ }^{1,2}$ \\ ${ }^{1}$ Department of Physics, Hokkaido University, 060-0810 Sapporo, Japan \\ ${ }^{2}$ Reaction Nuclear Data Centre, Faculty of Science, Hokkaido University, 060-0810 Sapporo, Japan
}

(Received 16 February 2017; published 22 June 2017)

\begin{abstract}
The decay properties of the linear-chain states in ${ }^{14} \mathrm{C}$ are investigated by using antisymmetrized molecular dynamics. The calculation predicts two rotational bands with linear-chain configurations having the $\pi$-bond and $\sigma$-bond valence neutrons. For the $\pi$-bond linear-chain, the calculated excitation energies and the widths of $\alpha$-decay to the ground state of ${ }^{10} \mathrm{Be}$ reasonably agree with the experimental candidates observed by the $\alpha+{ }^{10} \mathrm{Be}$ resonant scattering. On the other hand, the $\sigma$-bond linear-chain is the candidate of the higher-lying resonant states reported by the breakup reaction. As the evidence of the $\sigma$-bond linear chain, we discuss its decay pattern. It is found that the $\sigma$-bond linear chain not only decays to the excited band of ${ }^{10} \mathrm{Be}$ but also decays to the three-body channel of ${ }^{6} \mathrm{He}+\alpha+\alpha$, and the branching ratio of these decays are comparable. Hence, we suggest that this characteristic decay pattern is a strong signature of the linear-chain formation and a key observable to distinguish two different linear chains.
\end{abstract}

DOI: 10.1103/PhysRevC.95.064318

\section{INTRODUCTION}

Since the linear-chain configuration of $3 \alpha$ clusters (linearly aligned $3 \alpha$ clusters) was suggested in 1950s [1], many studies have been devoted to search it in the excited states of ${ }^{12} \mathrm{C}$ [2-10]. Nevertheless, no positive evidence has been obtained, and it is considered that the linear chain is unstable against the bending motion $[5,8]$.

Instead of ${ }^{12} \mathrm{C}$, neutron-rich $\mathrm{C}$ isotopes have attracted much interest in these decades as new candidates of the linear chain, because it is expected that the valence neutrons will play a gluelike role and stabilize the linear chain against the bending motion [11]. Theoretical studies predict the rotational bands with the linear-chain configurations in C isotopes [12-22]. It was pointed out that the motion of the valence neutron can be qualitatively interpreted in terms of the molecular orbits analogous to the Be isotopes [23-29], which are called $\pi$ and $\sigma$ orbits. Concurrently, rather promising candidates of the linear chain were reported in ${ }^{14} \mathrm{C}$ and ${ }^{16} \mathrm{C}$ by several experiments [30-38].

In our previous study [22], based on an antisymmetrized molecular dynamics (AMD) calculation, we pointed out that two positive-parity rotational bands in ${ }^{14} \mathrm{C}$ have the linear-chain configurations. The first band, which we call a $\pi$-bond linear chain, has two valence neutrons in a $\pi$ orbit and is built on the $0^{+}$state at $14.6 \mathrm{MeV}$ which is just above the $\alpha+{ }^{10} \mathrm{Be}$ threshold but below the $2 \alpha+{ }^{6} \mathrm{He}$ threshold. It was found that the energies and widths of the resonances observed by the $\alpha+{ }^{10}$ Be elastic scattering [36,37] qualitatively agree with the theoretical calculations $[15,18]$ including ours. Therefore, they are regarded as the $\pi$-bond linear-chain candidates, although the experimental spin-parity assignment was somewhat ambiguous. The other band, named the $\sigma$-bond linear chain, has valence neutrons in a $\sigma$ orbit and is built on the $0^{+}$state above the $\alpha+{ }^{10} \mathrm{Be}$ and $2 \alpha+{ }^{6} \mathrm{He}$ thresholds. It has a more elongated linear-chain configuration and larger moment of inertia than the former band, but the experimental counterpart was not known at that time.

Quite recently, very interesting data were reported by two groups. Yamaguchi et al. [39] reported the result of the $\alpha+{ }^{10} \mathrm{Be}$ elastic scattering and updated the information about the candidates of the $\pi$-bond linear chain. Although the spin-parity assignments for several states are not consistent with those found in previous experiments [35-37,39], they first observed a candidate of the bandhead of the linear chain $\left(J^{\pi}=0^{+}\right)$, which should be compared with theoretical calculations. The other experiment was reported by Tian et al. [40] and $\mathrm{Li}$ et al. [41] who observed the resonances populated by the ${ }^{9} \mathrm{Be}\left({ }^{9} \mathrm{Be}, \alpha+{ }^{10} \mathrm{Be}\right) \alpha$ reaction. In addition to the same resonances reported by Yamaguchi et al., they reported the decay properties of several resonances located above the $\alpha+{ }^{10} \mathrm{Be}$ and $2 \alpha+{ }^{6} \mathrm{He}$ thresholds. Based on the observed energies and decay pattern, these new resonances were suggested as the candidates of the $\sigma$-bond linear chain.

These new data motivated us to perform an additional analysis and to summarize the calculated and observed properties of the linear-chain bands in ${ }^{14} \mathrm{C}$. We investigated several decay modes of the linear-chain bands whose wave functions were obtained in our previous work [22]. By comparison with the new data, it is found that the agreement between the calculated and observed $\pi$-bond linear-chain band is plausible. It is also shown that the observed unique decay pattern of the resonances reported by Tian et al. is similar to that of the calculated $\sigma$-bond linear-chain band, which supports the existence of two different linear-chain bands with $\pi$ and $\sigma$ bonding. In addition to these analysis, it was found that the $\sigma$-bond linear chain decays to the ${ }^{6} \mathrm{He}+{ }^{8} \mathrm{Be}$ channel as well as the $\alpha+{ }^{10} \mathrm{Be}$ channel, and their branching ratios are comparable. Hence, we suggest that the sequential three-body decay of ${ }^{14} \mathrm{C}^{*} \rightarrow$ ${ }^{6} \mathrm{He}+{ }^{8} \mathrm{Be} \rightarrow{ }^{6} \mathrm{He}+\alpha+\alpha$ constitutes important evidence of the $\sigma$-bond linear chain.

The paper is organized as follows: The AMD framework and the method used to estimate the reduced widths amplitude for the $\alpha+{ }^{10} \mathrm{Be}$ and ${ }^{6} \mathrm{He}+{ }^{8} \mathrm{Be}$ decays are explained in the next section. In Sec. III, the excitation energies and decay widths of the linear-chain states are shown and compared with the observed data to suggest the assignment of the linear-chain bands. In the last section, we summarize this work. 


\section{THEORETICAL FRAMEWORK}

In this study, we analyze the wave functions of linear-chain states obtained in our previous work [22]. For the sake of self-containedness, in Sec. II A, we briefly explain how those wave functions were calculated. In Sec. II B and II C, we explain the method to evaluate the decay modes of linear-chain states used in the present study.

\section{A. Variational calculation and generator coordinate method}

We use the microscopic $A$-body Hamiltonian,

$$
\hat{H}=\sum_{i=1}^{A} \hat{t}_{i}-\hat{t}_{\mathrm{c} . \mathrm{m} .}+\sum_{i<j}^{A} \hat{v}_{i j}^{N}+\sum_{i<j}^{Z} \hat{v}_{i j}^{C},
$$

where the Gogny D1S interaction [42] is used as an effective nucleon-nucleon interaction $\hat{v}^{N}$. The Coulomb interaction $\hat{v}^{C}$ is approximated by a sum of seven Gaussians. The kinetic energy of the center-of-mass $\hat{t}_{\mathrm{c} . \mathrm{m}}$. is exactly removed.

The variational wave function $\Phi^{\Pi}$ is a parity-projected intrinsic wave function $\Phi_{\text {int }}$, and $\Phi_{\text {int }}$ is represented by a Slater determinant of single-particle wave packets,

$$
\begin{gathered}
\Phi^{\Pi}=\hat{P}^{\Pi} \Phi_{\text {int }}, \\
\Phi_{\text {int }}=\mathcal{A}\left\{\varphi_{1}, \varphi_{2}, \ldots, \varphi_{A}\right\},
\end{gathered}
$$

where $\hat{P}^{\Pi}$ denotes parity projector. In this study, we focus on the positive-parity states $(\pi=+) . \varphi_{i}$ is the single-particle wave packet which is a direct product of the deformed Gaussian spatial part [43], spin $\left(\chi_{i}\right)$, and isospin $\left(\xi_{i}\right)$ parts,

$$
\begin{gathered}
\varphi_{i}(\boldsymbol{r})=\phi_{i}(\boldsymbol{r}) \otimes \chi_{i} \otimes \xi_{i}, \\
\phi_{i}(\boldsymbol{r})=\exp \left\{-\sum_{\sigma=x, y, z} v_{\sigma}\left(r_{\sigma}-\frac{Z_{i \sigma}}{\sqrt{\nu_{\sigma}}}\right)^{2}\right\}, \\
\chi_{i}=a_{i} \chi_{\uparrow}+b_{i} \chi_{\downarrow}, \quad \xi_{i}=p \text { or } n .
\end{gathered}
$$

The centroids of the Gaussian wave packets, $\boldsymbol{Z}_{i}$, the direction of nucleon spin, $a_{i}, b_{i}$, and the width parameter of the deformed Gaussian, $v_{\sigma}$, are the variational parameters. The variational parameters are determined so that $E^{\Pi}$, which is a sum of the energy and constraint potential is minimized:

$$
E^{\Pi}=\frac{\left\langle\Phi^{\Pi}|\hat{H}| \Phi^{\Pi}\right\rangle}{\left\langle\Phi^{\Pi} \mid \Phi^{\Pi}\right\rangle}+v_{\beta}(\langle\beta\rangle-\beta)^{2}+v_{\gamma}(\langle\gamma\rangle-\gamma)^{2},
$$

where $\langle\beta\rangle$ and $\langle\gamma\rangle$ are the quadrupole deformation parameters of the intrinsic wave function defined in Ref. [44], and $v_{\beta}, v_{\gamma}$ are chosen to be a sufficiently large value. $E^{\Pi}$ is minimized by the frictional cooling method, and we obtain the optimized wave function $\Phi^{\Pi}(\beta, \gamma)=\hat{P}^{\Pi} \Phi_{\text {int }}(\beta, \gamma)$ which has the minimum energy for each set of $\beta$ and $\gamma$.

After the variational calculation, the eigenstate of the total angular momentum $J$ is projected out,

$$
\Phi_{M K}^{J \Pi}(\beta, \gamma)=\hat{P}_{M K}^{J} \Phi^{\Pi}(\beta, \gamma) .
$$

Here, $\hat{P}_{M K}^{J}$ is the angular-momentum projector. Next, we perform the generator coordinate method (GCM) calculation by employing the quadrupole deformation parameters $\beta$ and $\gamma$ as the generator coordinate. The wave function of GCM reads

$$
\Psi_{M n}^{J \Pi}=\sum_{i} \sum_{K} c_{K i n}^{J \Pi} \Phi_{M K}^{J \Pi}\left(\beta_{i}, \gamma_{i}\right)
$$

where the coefficients $c_{K i n}^{J \Pi}$ and eigenenergies $E_{n}^{J \Pi}$ are obtained by solving the Hill-Wheeler equation [45].

\section{B. Reduced width amplitude and decay width}

By using the GCM wave function, we estimate the reduced width amplitudes (RWA) $y_{l j_{n}^{\pi}}(r)$ for the $\alpha+{ }^{10} \mathrm{Be}$ and ${ }^{6} \mathrm{He}+$ ${ }^{8}$ Be decays, which are defined as

$$
y_{l j_{n}^{\pi}}(r)=\sqrt{\frac{A !}{A_{\mathrm{He}} ! A_{\mathrm{Be}} !}}\left\langle\phi_{\mathrm{He}}\left[\phi_{\mathrm{Be}}\left(j_{n}^{\pi}\right) Y_{l 0}(\hat{r})\right]_{J^{\Pi} M} \mid \Psi_{M n}^{J \Pi}\right\rangle,
$$

where $\phi_{\mathrm{He}}$ denotes the ground-state wave function for ${ }^{4} \mathrm{He}$ or ${ }^{6} \mathrm{He}$, and $\phi_{\mathrm{Be}}\left(j_{n}^{\pi}\right)$ denotes the wave functions for daughter nucleus ${ }^{10} \mathrm{Be}$ or ${ }^{8} \mathrm{Be}$ with spin-parity $j_{n}^{\pi} . Y_{l 0}(\hat{r})$ is the orbital angular momentum of the intercluster motion, and it is coupled with the angular momentum of $\operatorname{Be}\left(j_{n}^{\pi}\right)$ to yield the total spinparity $J^{\Pi} . A_{\mathrm{He}}$ and $A_{\mathrm{Be}}$ are the mass numbers of $\mathrm{He}$ and $\mathrm{Be}$, respectively. The reduced width $\gamma_{l j_{n}^{\pi}}$ is given by the square of the RWA,

$$
\gamma_{l j_{n}^{\pi}}^{2}(a)=\frac{\hbar^{2}}{2 \mu a}\left[a y_{l j_{n}^{\pi}}(a)\right]^{2},
$$

and the partial decay width is a product of the reduced width and the penetration factor $P_{l}(a)$,

$$
\Gamma_{l j_{n}^{\pi}}=2 P_{l}(a) \gamma_{l j_{n}^{\pi}}^{2}(a), \quad P_{l}(a)=\frac{k a}{F_{l}^{2}(k a)+G_{l}^{2}(k a)},
$$

where $a$ denote the channel radius, and $P_{l}$ is given by the Coulomb regular and irregular wave functions $F_{l}$ and $G_{l}$. The wave number $k$ is determined by the decay $Q$ value and the reduced mass $\mu$ as $k=\sqrt{2 \mu E_{Q}}$.

To reduce the computational cost, we employ an approximate method given in Ref. [46] to calculate Eq. (9). In this method the antisymmetrization effect is neglected by choosing sufficiently large intercluster distance $a$, and RWA is approximated by the overlap between the GCM wave function and the Brink-Bloch wave function $\Phi_{B B}^{J \pi j l}(a)$ in which He and Be clusters are placed with the intercluster distance $a$ as illustrated in Fig. 1,

$$
\begin{aligned}
\left|a y_{l j^{\pi^{\prime}}}(a)\right|^{2} & \simeq \sqrt{\frac{\gamma}{2 \pi}} \mid\left\langle\Phi_{B B}^{J \pi j l}(a)\left|\Psi_{M n}^{J \pi}\right|^{2},\right. \\
\gamma & =\frac{A_{\mathrm{He}} A_{\mathrm{Be}}}{A} v_{B B},
\end{aligned}
$$

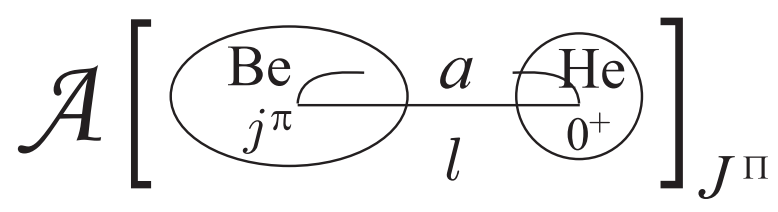

FIG. 1. Schematic figure showing the Brink-Bloch wave function. 


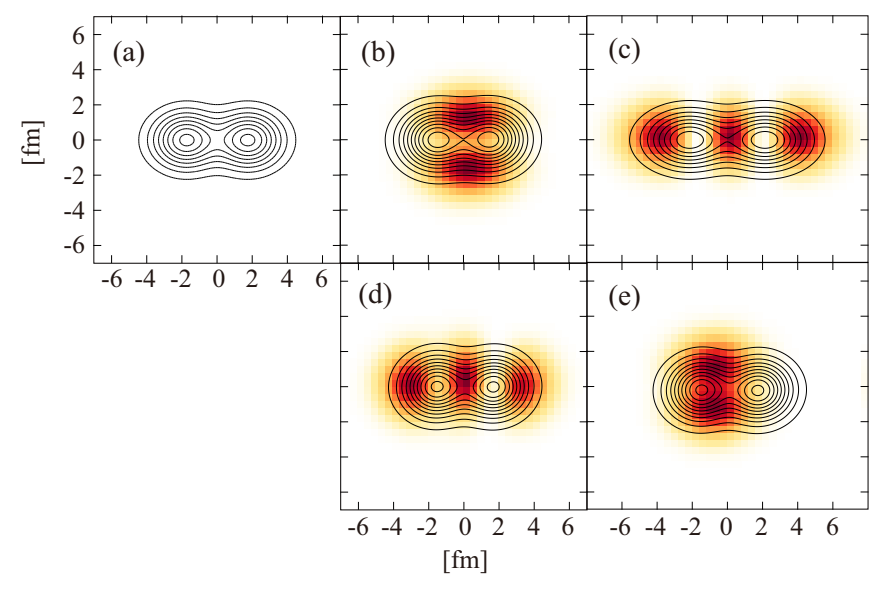

FIG. 2. The density distribution of (a) ${ }^{8} \mathrm{Be}$, the positive-parity states of ${ }^{10} \mathrm{Be}$ with valence neutrons in a (b) $\pi$ orbit and (c) $\sigma$ orbit, and (d), (e) negative-parity states of ${ }^{10} \mathrm{Be}$. The contour lines show the proton density distributions. The color plots in panels (b) and (c) show the single-particle orbits occupied by the most weakly bound neutron. In the negative-parity state, the color plots of panel (d) show the single-particle orbits occupied by the most weakly bound neutron, and those of panel (e) show the other valence neutron.

where $v_{B B}$ denotes the width parameter of the Gaussian wave packet of Brink-Bloch wave function.

In this study, the Brink-Bloch wave function is constructed as follows: First, the intrinsic wave functions for ${ }^{10} \mathrm{Be}$ and ${ }^{8} \mathrm{Be}$ denoted by $\psi_{\mathrm{Be}}$ are generated by the AMD energy variation. The intrinsic wave function of $\mathrm{Be}$ is approximated by a single AMD Slater determinant with spherical Gaussian wave packets with the width parameter $\nu_{B B}=0.16 \mathrm{fm}^{-2}$. In the case of ${ }^{8} \mathrm{Be}$, the wave functions of the $0_{1}^{+}$and $2_{1}^{+}$states are calculated by the bound-state approximation. The density distribution of obtained intrinsic wave function of ${ }^{8} \mathrm{Be}$ is shown in Fig. 2(a) in which the distance between two $\alpha$ clusters is approximately 3.4 $\mathrm{fm}$. For the positive-parity states of ${ }^{10} \mathrm{Be}$, we obtained the two different intrinsic wave functions shown in Figs. 2(b) and 2(c) in which two valence neutrons occupy so-called $\pi$ and $\sigma$ orbits, respectively. We regard that the former correspond to the ground band (the $0_{1}^{+}, 2_{1}^{+}$, and $4_{1}^{+}$states), while the latter is the excited band (the $0_{2}^{+}, 2_{3}^{+}$, and $4_{2}^{+}$states). For the negative-parity states of ${ }^{10} \mathrm{Be}$, we obtain an intrinsic wave function shown in Figs. 2(d) and 2(e) in which one of two valence neutrons occupies a $\pi$ orbit and the other occupies a $\sigma$ orbit. Then, they are projected to the eigenstate of the angular momentum $j^{\pi}$ as $\phi_{\mathrm{Be}}\left(j_{n}^{\pi}\right)=\hat{P}_{m k}^{j \pi} \psi_{\mathrm{Be}}$, where we approximate that the Be wave functions are the eigenstates of the angular momentum $\hat{j}_{z}$ in the intrinsic frame. The eigenvalue $k$ is assumed to be $k=0$ in the positive-parity states and $k=-1$ in the negative-parity states. The construction of the wave function of ${ }^{4} \mathrm{He}$ and ${ }^{6} \mathrm{He}$ is explained in the next section. The Brink-Bloch wave function is constructed by placing these $\mathrm{He}$ and $\mathrm{Be}$ wave functions with the intercluster distance $a$,

$$
\Phi_{B B}^{j m}(a)=\mathcal{A}\left\{\phi_{\mathrm{He}}\left(-\frac{A_{\mathrm{Be}}}{A} a\right) \hat{P}_{m k}^{j} \psi_{\mathrm{Be}}\left(\frac{A_{\mathrm{He}}}{A} a\right)\right\},
$$

and projected to the eigenstate of the total spin-parity $J^{\Pi}$ as $\hat{P}_{M m}^{J^{\Pi}} \Phi_{B B}^{j m}(a)$. Then, we construct the wave function, in which the angular momentum $l$ of the intercluster motion and the angular momentum $j$ of Be are coupled to the total spin-parity $J^{\Pi}$, by summing up over all possible values of $m$,

$$
\Phi_{B B}^{J \pi j l}(a)=n \frac{2 l+1}{2 J+1} \sum_{m} C_{l 0 j m}^{J m} \hat{P}_{M m}^{J^{\pi}} \Phi_{B B}^{j m}(a),
$$

where $C_{l 0 j m}^{J m}$ and $n$ denotes the Clebsch-Gordan coefficient and the normalization factor.

Generally, the partial decay width should be independent on the choice of the channel radius. However, in the practical calculation, the channel radius must be properly chosen to stabilize the results because of the following two problems: First, the channel radius should not be too large a value, because we adopt the bound-state approximation in the GCM calculation and hence the wave function is not correct at large intercluster distance. Second, the channel radius $a$ should not be too small, because the antisymmetrization effect cannot be neglected and the approximation is not valid. Therefore, we adopted two different values for the channel radius. The first choice is $a=5.2 \mathrm{fm}$ which is common to the value used in the $R$-matrix analysis of the $\pi$-bond linear-chain candidates observed in Ref. [36] and close to that in Ref. [39]. Unfortunately, this choice of channel radius was found inappropriate for the analysis of the $\sigma$-bond linear chain. Because the $\sigma$-bond linear chain is dominated by the ${ }^{10} \mathrm{Be}\left(0_{2}^{+}, 2_{3}^{+}\right)+\alpha$ channels and ${ }^{10} \mathrm{Be}\left(0_{2}^{+}, 2_{3}^{+}\right)$have larger radii than the ground state, the larger channel radius should be used to avoid the antisymmetrization effect. Hence, we used $a=6.0$ fm for the analysis of the $\sigma$-bond linear chain.

\section{C. ${ }^{6}$ He reduced width amplitude}

Here, we explain how the wave functions of ${ }^{4} \mathrm{He}$ and ${ }^{6} \mathrm{He}$ clusters are constructed. The wave function of ${ }^{4} \mathrm{He}$ is approximated by a $\left(0 s_{1 / 2}\right)^{4}$ wave function of a harmonic oscillator (H.O.) which is represented by the Gaussian wave packet with the width of $v_{B B}=0.16 \mathrm{fm}^{-2}$,

$$
\phi_{0 s}(\boldsymbol{r})=(2 v / \pi)^{3 / 4} e^{-v r^{2}} \otimes \chi
$$

The ground state of ${ }^{6} \mathrm{He}$ is approximated by a $\left(0 s_{1 / 2}\right)^{4}\left(0 p_{3 / 2}\right)^{2}$ configuration as

$$
\phi_{\mathrm{He}}^{J=0}=\mathcal{A}\left\{\left(0 s_{1 / 2}\right)^{4}\left[0 p_{3 / 2} \otimes 0 p_{3 / 2}\right]^{J=0}\right\},
$$

where $0 p_{3 / 2}$ is also the eigenfunction of the H.O. In the practical calculation, we do not use H.O. wave functions directly, but the $0 p_{3 / 2}$ wave function is represented by the sum of the infinitesimally shifted Gaussian wave packets $\phi_{0 s}(\boldsymbol{r} ; \boldsymbol{\epsilon})=(2 v / \pi)^{3 / 4} \exp -v\left(\boldsymbol{r}-\boldsymbol{\epsilon}^{2}\right) \otimes \chi$. This greatly reduces the computational cost because it is possible to use ordinary computational code for AMD to calculate Eq. (12). The relationship between the shifted Gaussian wave packets and the H.O. wave function is given as follows:to first order in 
the shift $\epsilon$,

$$
\begin{aligned}
\phi_{0 s}(\boldsymbol{r} ; \boldsymbol{\epsilon})-\phi_{0 s}(\boldsymbol{r} ; \mathbf{0})= & \left(\frac{2 v}{\pi}\right)^{3 / 4}\left\{e^{-v(\boldsymbol{r}-\boldsymbol{\epsilon})^{2}}-e^{-v r^{2}}\right\} \\
& \times \otimes\left(a \chi_{\uparrow}+b \chi_{\downarrow}\right) \\
\simeq & \left(\frac{2 v}{\pi}\right)^{3 / 4} 2 v \boldsymbol{r} \cdot \boldsymbol{\epsilon} e^{-v r^{2}} \otimes\left(a \chi_{\uparrow}+b \chi_{\downarrow}\right) \\
= & -\left(\frac{2 v}{\pi}\right)^{3 / 4} 2 v r e^{-v r^{2}}\left[a \mathcal{Y}_{1-1}(\boldsymbol{\epsilon}) \phi_{p_{3 / 2} 3 / 2}\right. \\
& +b \mathcal{Y}_{11}(\boldsymbol{\epsilon}) \phi_{p_{3 / 2}-3 / 2} \\
& +\sqrt{\frac{1}{3}}\left\{b \mathcal{Y}_{1-1}(\boldsymbol{\epsilon})-\sqrt{2} a \mathcal{Y}_{10}(\boldsymbol{\epsilon})\right\} \phi_{p_{3 / 2} 1 / 2} \\
& +\sqrt{\frac{1}{3}}\left\{a \mathcal{Y}_{11}(\boldsymbol{\epsilon})-\sqrt{2} b \mathcal{Y}_{10}(\boldsymbol{\epsilon})\right\} \phi_{p_{3 / 2}-1 / 2} \\
& +\sqrt{\frac{1}{3}}\left\{\sqrt{2} b \mathcal{Y}_{1-1}(\boldsymbol{\epsilon})+a \mathcal{Y}_{10}(\boldsymbol{\epsilon})\right\} \phi_{p_{1 / 2} 1 / 2} \\
& -\sqrt{\frac{1}{3}}\left\{\sqrt{2} a \mathcal{Y}_{11}(\boldsymbol{\epsilon})+b \mathcal{Y}_{10}(\boldsymbol{\epsilon})\right\} \\
& \left.\times \phi_{p_{1 / 2}-1 / 2}\right],
\end{aligned}
$$

where $\mathcal{Y}_{1 m}(\boldsymbol{r})$ is the regular solid spherical harmonics,

$$
\mathcal{Y}_{1 m}(\boldsymbol{r})=\sqrt{\frac{4 \pi}{3}} r Y_{1 m}(\hat{r}),
$$

where $\phi_{p_{3 / 2} m}$ and $\phi_{p_{1 / 2 m}}$ denote the $0 p_{3 / 2}$ and $0 p_{1 / 2}$ wave functions with the magnetic quantum number $m$. From Eq. (17), we see that $0 p_{3 / 2}$ wave functions can be described by the sum of the $\phi_{0 s}(\boldsymbol{r} ; \boldsymbol{\epsilon})$ with proper choice of $a, b$, and $\boldsymbol{\epsilon}$. Thus, Eq. (16) is represented by the sum of the Slater determinant of the shifted Gaussian packets. In the practical calculation the magnitude of $\epsilon$ is chosen as $|\boldsymbol{\epsilon}|=0.02$.

\section{RESULTS AND DISCUSSION}

In Sec. III A, we summarize the properties of the $\pi$-bond and $\sigma$-bond linear chains studied in our previous work [22]. In Secs. III B and III C, by referring the latest experimental data and the theoretical analysis of the decay modes, we discuss the assignment of the linear-chain bands.

\section{A. Calculated linear-chain bands}

Figure 3 summarizes the calculated rotational bands with the linear-chain configurations presented in Ref. [22] and experimental data $[36,37,39,40]$. The $\pi$-bond linear-chain band shown by blue squares is built on the $0^{+}$state at $14.6 \mathrm{MeV}$ which lies just above the $\alpha+{ }^{10} \mathrm{Be}\left(0_{1}^{+}\right)$threshold but below the $\alpha+{ }^{10} \mathrm{Be}\left(0_{2}^{+}\right)$and ${ }^{6} \mathrm{He}+{ }^{8} \mathrm{Be}\left(0^{+}\right)$thresholds. The other band, the $\sigma$-bond linear chain, is built on the $0^{+}$state at $22.2 \mathrm{MeV}$ which is above all of those thresholds.

Theoretically, the assignment of these two bands is rather unique. The reasons for the assignment and the properties

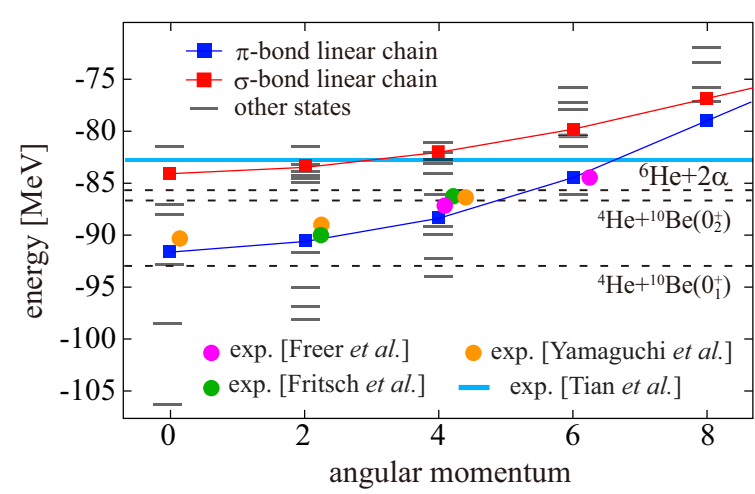

FIG. 3. The positive-parity energy levels up to $J^{\pi}=8^{+}$. Filled circles show the resonances with the definite spin-parity assignments observed in the $\alpha+{ }^{10} \mathrm{Be}$ resonant scattering [36,37,39]. Blue line shows the resonance at $22.5 \mathrm{MeV}$ without spin-parity assignment observed in the breakup reaction [41]. Filled boxes show the calculated linear-chain bands, while lines show the other states. Only even angular momenta are shown.

of the linear-chain bands are as follows: First, these bands are dominated by the intrinsic states having the linear-chain configurations shown in Fig. 4. The $\pi$-bond linear chain has large overlap with the intrinsic wave function shown in Fig. 4(a), which amounts to $87 \%$ for the $0^{+}$state at $14.6 \mathrm{MeV}$. The proton density distribution shown by solid lines clearly indicates the formation of the linearly aligned three-alpha clusters surrounded by the two valence neutrons shown by the color plot. As already discussed in Refs. [19,22], in terms of the molecular orbit picture, this valence neutron orbit is interpreted as the $\pi$ orbit which is composed of the perpendicular alignment of the $p$ wave around the alpha cluster, as illustrated in Fig. 5(a). The $\sigma$-bond linear chain is dominated by the intrinsic wave function shown in Fig. 4(b), whose overlap with the $0^{+}$state at $22.2 \mathrm{MeV}$ amounts to $99 \%$. Again, we recognize the formation of the $3 \alpha$ linear chain, but the valence neutron orbit is different. It is interpreted as the $\sigma$ orbit shown in Fig. 5(b) which is composed of the parallel alignment of the $p$ orbits. Since all other states in this energy region denoted by lines in Fig. 3 have much less

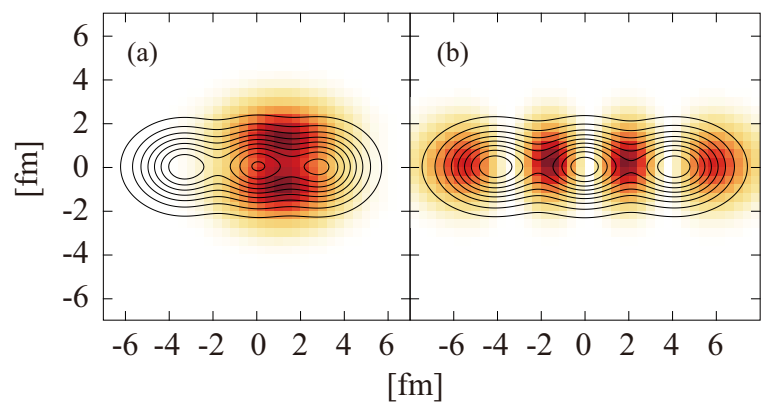

FIG. 4. The density distribution of the linear-chain configurations of ${ }^{14} \mathrm{C}$, with valence neutrons in a (a) $\pi$ orbit and (b) $\sigma$ orbit. The contour lines show the proton density distributions. The color plots show the single-particle orbits occupied by the most weakly bound neutron. 
(a)

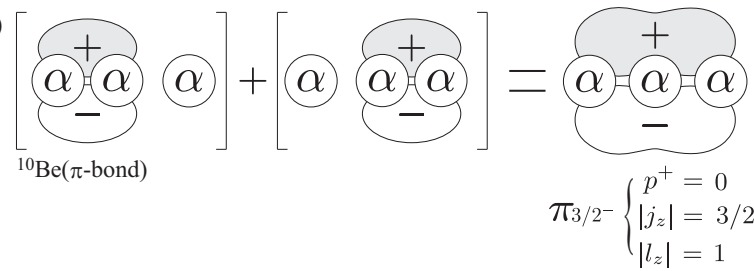

(b)

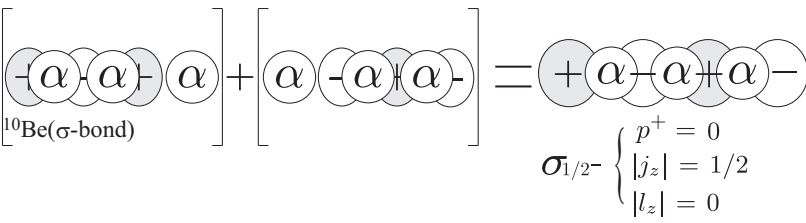

(c)

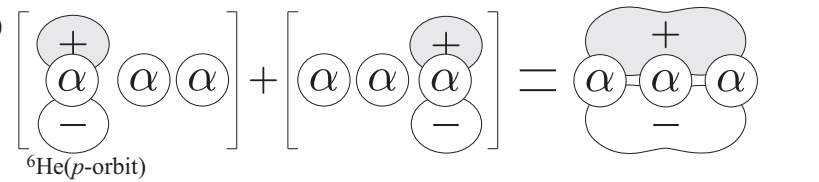

(d)

${ }^{6} \mathrm{He}(p$-orbit $)$

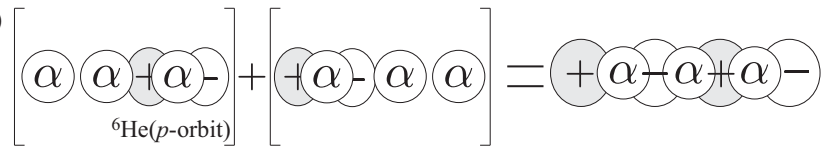

FIG. 5. Schematic figure showing the $\pi$ and $\sigma$ orbits around the linear chain. The combination of the $p$ orbits perpendicular to the symmetry axis generates $\pi$ orbits, while the combination of parallel orbits generates $\sigma$ orbit.

overlap with the configurations, the linear-chain bands can be clearly assigned.

Second, the $B(E 2)$ transition strengths between the member states of these bands are rather strong compared with other inband and interband transitions as listed in Table III in Ref. [22], which is consistent with the dominance of the strongly deformed intrinsic shapes with linear-chain configuration. Because the $\sigma$-bond linear chain is more strongly deformed than the $\pi$-bond linear chain, its in-band transition strengths are stronger than those of the $\pi$-bond linear chain. Deference of the deformation is also reflected to the moment of inertia; $\hbar / 2 \Im=179$ and $98 \mathrm{keV}$ for $\pi$-bond and $\sigma$-bond linear-chain bands, respectively.

Finally, among the excited states located around $\mathrm{z} \alpha+$ ${ }^{10} \mathrm{Be}\left(0_{1}^{+}\right), \alpha+{ }^{10} \mathrm{Be}\left(0_{2}^{+}\right)$, and ${ }^{6} \mathrm{He}+{ }^{8} \mathrm{Be}\left(0^{+}\right)$thresholds, the linear-chain bands have largest $\alpha$ and ${ }^{6} \mathrm{He}$ reduced widths. Therefore, the alpha decaying resonances in the vicinity of these thresholds are, if observed, regarded as the candidates of the linear-chain bands.

\section{B. Resonances observed in $\alpha+{ }^{10}$ Be scattering and assignment of $\pi$-bond linear-chain band}

Now, we discuss the assignment of the linear-chain bands based on the latest experimental data. Freer et al. [36], Fritsch et al. [37], and more recently Yamaguchi et al. [39] independently reported the resonances observed in $\alpha+{ }^{10} \mathrm{Be}$ scattering, which are shown by circles in Fig. 3 and summarized in Table I. Freer et al. reported the $4^{+}$and $6^{+}$resonances at 18.22 and $20.80 \mathrm{MeV}$, respectively, while Fritsch et al. reported the $2^{+}$and $4^{+}$resonances at 15.0 and $19.0 \mathrm{MeV}$. A candidate of the $2^{+}$resonance at $17.95 \mathrm{MeV}$ was also reported by Freer $e$ al. but is not shown in Fig. 3 because the spin-parity assignment is not so firm. Yamaguchi et al. reported the $0^{+}$, $2^{+}$, and $4^{+}$resonances at $15.07,16.22$, and $18.87 \mathrm{MeV}$. The $4^{+}$energy is very close to that observed by Fritsch $e t$ al. and the $0^{+}$state may correspond to the $15.0 \mathrm{MeV}$ state of Fritsch et al., which was assigned as $2^{+}$. We also note that the same resonances were also observed in the breakup [47] and transfer reactions [40,41], although the spin-parity assignment was not given.

Although they suggest different spin-parity assignments, we consider that they observed essentially the same resonances which are assigned to the $\pi$-bond linear-chain band from the following reasons. First, it is clear that these resonance energies very nicely agree with those of the calculated $\pi$-bond linear chain, regardless of the spin-parity assignments. Furthermore, the observed data show the large moment of inertia of the band; $\hbar / 2 \Im=116$ [36] and $190 \mathrm{keV}$ [39]. In any cases, the very large moment of inertia are consistent with the large deformation of the linear-chain band which reaches to $3: 1$ ratio of the deformation axes. In particular, the moment of inertia reported by Yamaguchi et al. $(\hbar / 2 \Im=190 \mathrm{keV})$ is very close to the present result. However, it is reminded that there is an experimental uncertainty in the assignment of the $15.0 \mathrm{MeV}$ state $\left(J^{\pi}=0^{+}\right.$or $\left.2^{+}\right)$. Since the experimental spinparity assignment of the broad resonances is not easy, it should be carefully discussed because it strongly affects the discussion of the moment of inertia.

Second, as listed in Table I, the observed resonances have large alpha decay widths to the $\alpha+{ }^{10} \mathrm{Be}\left(0_{1}^{+}\right)$channel comparable with those of the calculated $\pi$-bond linear chain. Unfortunately, experimental data are not quantitatively consistent with each other. In particular, the alpha-decay widths reported by Yamaguhi et al. [39] are getting smaller with the increase of $J$ much faster than the calculated ones, while those reported in Refs. [36,37] are getting larger. Nevertheless, one

TABLE I. Excitation energies (MeV) and $\alpha$-decay widths $(\mathrm{keV})$ up to $J^{\pi}=8^{+}$of the linear-chain states and the experiments. $\Gamma_{\alpha}$ shows the decay to the ground state $\left(0_{1}^{+}\right)$of ${ }^{10} \mathrm{Be}$. Numbers in parentheses are tentative.

\begin{tabular}{|c|c|c|c|c|c|c|c|c|c|c|c|}
\hline$J^{\pi}$ & $E_{x}$ & $\Gamma_{\alpha}(5.2 \mathrm{fm})$ & $\Gamma_{\alpha}(6.0 \mathrm{fm})$ & $E_{x}$ & $\Gamma_{\alpha}(6.0 \mathrm{fm})$ & $E_{x}$ & $\Gamma_{\alpha}$ & $E_{x}$ & $\Gamma_{\alpha}$ & $E_{x}$ & $\Gamma_{\alpha}$ \\
\hline $2^{+}$ & 15.73 & 214 & 188 & 22.93 & 0.4 & (17.95) & $(760)$ & 15.0 & 290 & 16.22 & 190 \\
\hline $4^{+}$ & 17.98 & 149 & 147 & 24.30 & 0.3 & 18.22 & 200 & 19.0 & 340 & 18.87 & 45 \\
\hline $6^{+}$ & 21.80 & 123 & 151 & 26.45 & 0.2 & 20.80 & 300 & & & & \\
\hline
\end{tabular}


TABLE II. Excitation energies (MeV) and $\alpha$-decay widths (keV) to the $2_{1}^{+}$state of ${ }^{10} \mathrm{Be}$.

\begin{tabular}{ccccccc}
\hline \hline & \multicolumn{3}{c}{$\pi$-bond linear chain } & & \multicolumn{2}{c}{$\sigma$-bond linear chain } \\
\cline { 2 - 3 }$J^{\pi}$ & $E_{x}$ & $\Gamma_{\alpha}(5.2 \mathrm{fm})$ & $\Gamma_{\alpha}(6.0 \mathrm{fm})$ & & $E_{x}$ & $\Gamma_{\alpha}(6.0 \mathrm{fm})$ \\
\hline $0^{+}$ & 14.64 & & & 22.16 & 0.6 \\
$2^{+}$ & 15.73 & & & 22.93 & 0.2 \\
$4^{+}$ & 17.98 & 118 & 111 & & 24.30 & 1.8 \\
$6^{+}$ & 21.80 & 256 & 271 & & 26.45 & 0.4 \\
$8^{+}$ & 27.25 & 397 & 421 & & 29.39 & 0.8 \\
\hline \hline
\end{tabular}

would conclude that most of them are a few hundred $\mathrm{keV}$, which is the same order of magnitude as the calculated $\pi$-bond linear chain. This is an evidence of the linear-chain formation, because theories predict no other states than the $\pi$-bond linear-chain states which have large alpha decay widths in this energy region. It must be noted that the $\sigma$-bond linear-chain band has rather small decay widths to the $\alpha+{ }^{10} \operatorname{Be}\left(0_{1}^{+}\right)$ channel, which distinguishes the $\sigma$-bond linear chain from the $\pi$-bond linear chain. The reason for this decay suppression is explained in the next section.

Finally, theories predicted the decay of the $\pi$-bond linear chain to the $\alpha+{ }^{10} \mathrm{Be}\left(2_{1}^{+}\right)$channel despite of the smaller decay $Q$ value (Table II). This is because of the strong admixture of the $\alpha+{ }^{10} \mathrm{Be}\left(0_{1}^{+}\right)$and $\alpha+{ }^{10} \mathrm{Be}\left(2_{1}^{+}\right)$configurations in the $\pi$-bond linear chain, which originates in the strong coupling nature of the linearly aligned alpha clusters. Experimentally, the width of the $\alpha+{ }^{10} \mathrm{Be}\left(2_{1}^{+}\right)$decay has not been measured, but Fritsch et al. reported the decay of the $4^{+}$resonance to the $\alpha+{ }^{10} \mathrm{Be}\left(2_{1}^{+}\right)$channel. Thus, the excitation energies, moment of inertia, and the decay widths are consistent between theory and the $\alpha+{ }^{10} \mathrm{Be}$ scattering experiment and, thus, the formation of $\pi$-bond linear chain in ${ }^{14} \mathrm{C}$ looks rather plausible. However, it must be emphasized that further experimental studies are in need to resolve the inconsistency between the data and to firmly establish the linear-chain formation. In particular, the following points are essential and indispensable: (1) the assignment of the $15.0 \mathrm{MeV}$ state, $J^{\pi}=0^{+}$or $2^{+}$; (2) quantitative agreement of the resonance widths between the experimental data and also with the theoretical results; (3) the confirmation of the decays to the inelastic channels and quantitative evaluation of the partial decay widths.

\section{Higher-lying resonances observed in breakup reaction and the assignment of $\sigma$-bond linear-chain band}

In addition to the candidates of the $\pi$-bond linear chain, several alpha-decaying resonances are reported above the $\alpha+{ }^{10} \mathrm{Be}\left(0_{2}^{+}\right)$and ${ }^{6} \mathrm{He}+{ }^{8} \mathrm{Be}\left(0_{1}^{+}\right)$thresholds $[31,35,40,41]$. In particular, quite recently, Li et al. [41] reported the decay property of a resonance located at $22.5 \mathrm{MeV}$, which provides new insight into the structure of the resonance. Since the spin-parity of the $22.5 \mathrm{MeV}$ resonance was not assigned, it is shown by blue line in Fig. 3. We see that its energy is close to the calculated $0^{+}, 2^{+}, 4^{+}$, and $6^{+}$states of the $\sigma$-bond linear chain, although we cannot exclude the assignment to the $6^{+}$or $8^{+}$states of the $\pi$-bond linear chain.
To identify the structure of these resonances, we focus on the decay patterns of the $\pi$ - and $\sigma$-bond linear chains. The reduced widths to various decay channels summarized in Fig. 6 suggests unique decay patterns of the linear chains. From Figs. 6(a) to 6(d), we see that all of the $\pi$-bond linear-chain states decay to the ground band of ${ }^{10} \mathrm{Be}\left(0_{1}^{+}\right.$and $\left.2_{1}^{+}\right)$, but not to the excited band $\left(0_{2}^{+}\right.$and $\left.2_{3}^{+}\right)$. On the other hand, the $\sigma$-bond linear chain has quite the opposite pattern; it decays to the excited band, but not to the ground band. This clearly distinguishes two linear chains, and the reason of the difference is qualitatively understood from the intrinsic density distributions of the ${ }^{10} \mathrm{Be}$ and linear chains shown in Figs. 2 and 4 . Both the ground band of ${ }^{10} \mathrm{Be}$ and the $\pi$-bond linear chain has $\pi$-bonding neutrons and, hence, the $\pi$-bond linear chain can be described by the linear alignment of the ${ }^{10} \mathrm{Be}\left(0_{1}^{+}\right.$ and $2_{1}^{+}$) and alpha cluster as illustrated in Fig. 5(a). Since this configuration is orthogonal to the $\alpha+{ }^{10} \mathrm{Be}\left(0_{1}^{+}\right.$and $\left.2_{1}^{+}\right)$the decay suppression to the $\alpha+{ }^{10} \mathrm{Be}\left(0_{1}^{+}\right.$and $\left.2_{1}^{+}\right)$channels can be naturally understood. In the same way, the $\sigma$-bond linear chain can be described by the linear alignment of the ${ }^{10} \mathrm{Be}\left(0_{2}^{+}\right.$ and $\left.2_{3}^{+}\right)+\alpha$ as shown in Fig. 5(b) which explains the decay pattern of the $\sigma$-bond linear chain.

Experimentally, it has been reported that several resonances above $20 \mathrm{MeV}$ primary decay to the excited states of ${ }^{10} \mathrm{Be}$ around $6 \mathrm{MeV}$ [31,35,40,41] (see Table I of Ref. [41] for the summary of the experimental data). In particular, Li et al. [41] reported the relative branching ratio of some resonances and showed that the $22.5 \mathrm{MeV}$ resonance has approximately three times larger branching ratio to the $6 \mathrm{MeV}$ state of ${ }^{10} \mathrm{Be}$ than those to the ${ }^{10} \mathrm{Be}\left(0_{1}^{+}\right)$and ${ }^{10} \mathrm{Be}\left(2_{1}^{+}\right)$. Unfortunately, the experiment does not have sufficient energy resolution to identify the ${ }^{10} \mathrm{Be} 6 \mathrm{MeV}$ state out of the $0_{2}^{+}(6.179 \mathrm{MeV}), 2_{2}^{+}$ (5.958 MeV), $1_{1}^{-}(5.959 \mathrm{MeV})$, and $2_{1}^{-}(6.263 \mathrm{MeV})$ states. But, if we assume that the $6 \mathrm{MeV}$ state is the ${ }^{10} \mathrm{Be}\left(0_{2}^{+}\right)$, we consider that the decay pattern of the $22.5 \mathrm{MeV}$ resonance is similar to the calculated $\sigma$-bond linear chain. To illustrate it, we calculated the decay of the linear chains to the ${ }^{10} \mathrm{Be}\left(0_{1}^{+}\right)$, ${ }^{10} \mathrm{Be}\left(2_{1}^{+}\right)$, and excited states around $6 \mathrm{MeV}$. The decay to the ${ }^{10} \mathrm{Be}\left(2_{2}^{+}\right)$was not calculated because the triaxial deformation of ${ }^{10} \mathrm{Be}\left(2_{2}^{+}\right)$[27] demands much computational cost. But, we expect that the decay to the ${ }^{10} \mathrm{Be}\left(2_{2}^{+}\right)$should be negligible because its neutron configuration mismatches with the $\pi$ - and $\sigma$-bond linear chains. Table III summarizes the calculated decay widths of the linear-chain states around $22.5 \mathrm{MeV}$. We see that the calculated $\sigma$-bond linear-chain states have much larger decay width to ${ }^{10} \mathrm{Be}\left(0_{2}^{+}\right)$than to the ${ }^{10} \mathrm{Be}\left(0_{1}^{+}\right)$ and ${ }^{10} \mathrm{Be}\left(2_{1}^{+}\right)$in order of magnitude. On the contrary, the $\pi$-bond linear-chain states primarily decay to ${ }^{10} \mathrm{Be}\left(0_{1}^{+}\right)$and ${ }^{10} \mathrm{Be}\left(2_{1}^{+}\right)$, which contradicts observation. Thus the $22.5 \mathrm{MeV}$ state is a good candidate of the $\sigma$-bond linear chain. Of course, it must be emphasized that further experimental and theoretical studies are required to establish the $\sigma$-bond linear chain. For example, the measurement of the absolute value of the partial decay widths is essential. Theoretically, the admixture of the $\pi$ - and $\sigma$-bond configurations in ${ }^{10} \mathrm{Be}\left(0_{1}^{+}\right)$ and ${ }^{10} \mathrm{Be}\left(0_{2}^{+}\right)$should be properly considered, as it quantitatively affects the decay branching ratio of the linear chains. 

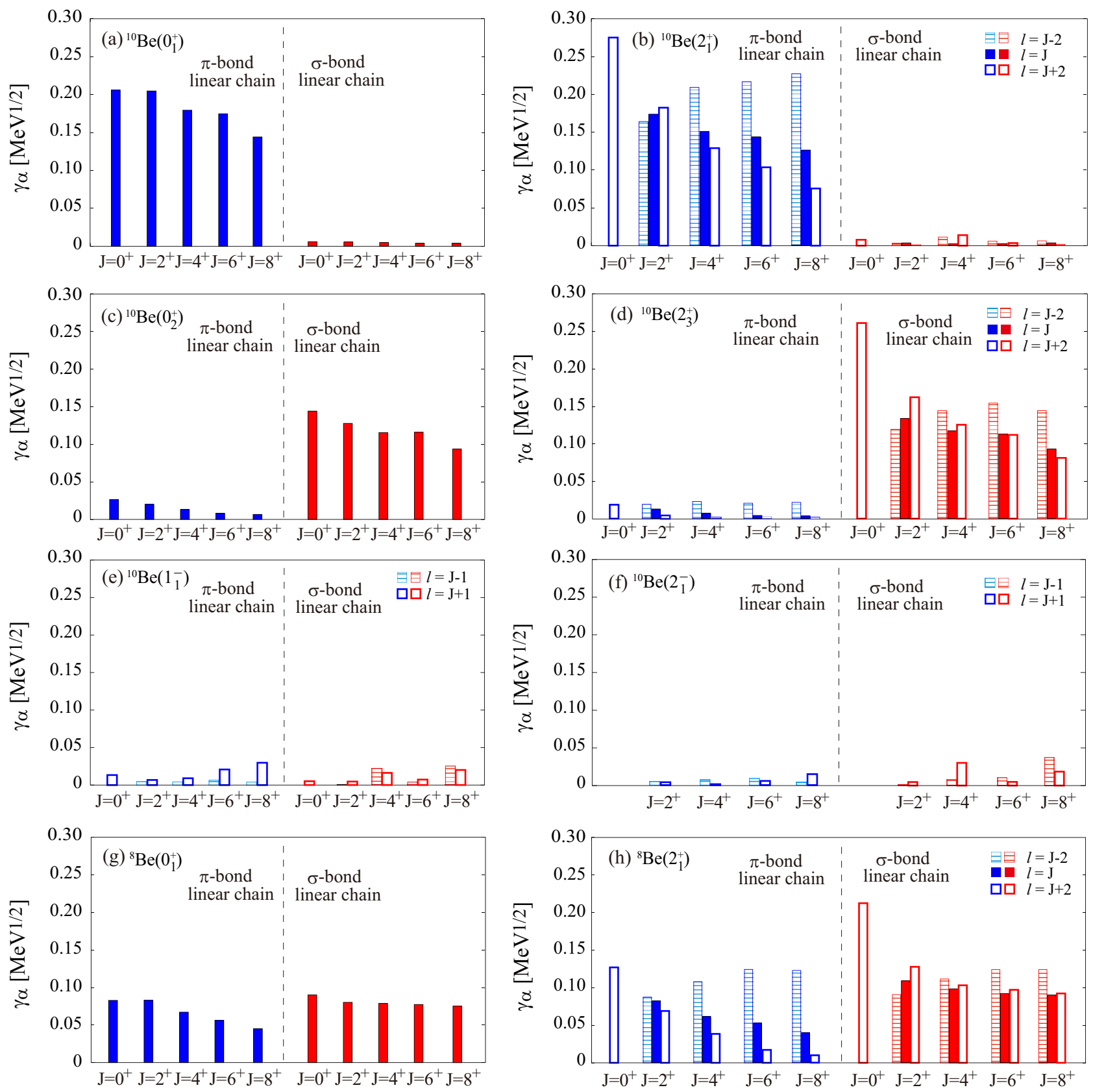

FIG. 6. Calculated $\alpha$-and ${ }^{6} \mathrm{He}$-decay reduced widths up to $J^{\pi}=8^{+}$. Panels (a) and (b) show the decay to the ground band of ${ }^{10} \mathrm{Be}(\pi$-bonded ${ }^{10} \mathrm{Be}$ ). Panels (c) and (d) show the decay to the excited band of ${ }^{10} \mathrm{Be}\left(\sigma\right.$-bonded $\left.{ }^{10} \mathrm{Be}\right)$. Panels (e) and (f) show the decay to the negative-parity states of ${ }^{10} \mathrm{Be}$. Panels $(\mathrm{g})$ and $(\mathrm{h})$ show the decay to the ground band of ${ }^{8} \mathrm{Be}$. The $l$ denotes the relative motion between $\mathrm{Be}\left(2^{+}\right)$and $\alpha$ particle. The channel radius $a$ is $6.0 \mathrm{fm}$.

Finally, we discuss the decays to ${ }^{6} \mathrm{He}+{ }^{8} \mathrm{Be}$ and ${ }^{5} \mathrm{He}+{ }^{9} \mathrm{Be}$ channels. Figures $6(\mathrm{~g})$ and $6(\mathrm{~h})$ show that both of the $\pi$ and $\sigma$-bond linear chains have large reduced widths in the ${ }^{6} \mathrm{He}+{ }^{8} \mathrm{Be}$ channel, which is another interesting feature of the linear chains. This is again schematically understood from Fig. 5. Because the valence neutron in $\pi$ and $\sigma$ orbits are covalent, the linear chains can also be described by the linear alignment of ${ }^{6} \mathrm{He}+{ }^{8} \mathrm{Be}$ as illustrated in Figs. 5(c) and 5(d). Therefore, the $\sigma$-bond linear-chain and high-spin states $\left(J^{\Pi} \geqslant 6^{+}\right)$of the $\pi$-bond linear chain which are located above the ${ }^{6} \mathrm{He}+{ }^{8} \mathrm{Be}$ threshold should also decay to the three-body final state through the sequential two body decays, ${ }^{14} \mathrm{C}^{*} \rightarrow{ }^{6} \mathrm{He}+{ }^{8} \mathrm{Be} \rightarrow{ }^{6} \mathrm{He}+\alpha+\alpha$. As listed in Table III, the decay widths of the $\sigma$-bond linear chain to the ${ }^{6} \mathrm{He}+{ }^{8} \mathrm{Be}$ channel are in the same order as those to the $\alpha+{ }^{10} \mathrm{Be}\left(0_{2}^{+}\right)$ channel, and hence, the decay to ${ }^{6} \mathrm{He}+\alpha+\alpha$ is more evidence of the linear-chain formation. We also comment on the decay to the ${ }^{5} \mathrm{He}+{ }^{9} \mathrm{Be}$ channel $\left({ }^{4} \mathrm{He}+n+{ }^{9} \mathrm{Be}\right)$ whose decay threshold is located very close to that of the ${ }^{6} \mathrm{He}+{ }^{8} \mathrm{Be}$ channel. Theoretically, the decay-width calculation for the odd-mass daughter nuclei demands much computational time and, thus, it was not performed in the present study. Nevertheless, we 
TABLE III. Partial decay widths $(\mathrm{keV})$ in six different channels for (a) the $\sigma$-bond linear-chain states and (b) $J^{\pi}=6^{+}, 8^{+}$states of the $\pi$-bond linear chain. The channel radius $a$ is $6.0 \mathrm{fm}$.

(a) $\sigma$-bond linear chain

\begin{tabular}{|c|c|c|c|c|c|c|c|}
\hline$J^{\pi}$ & $E_{x}$ & $\Gamma\left({ }^{10} \mathrm{Be}\left(0_{1}^{+} ; \pi^{2}\right)\right)$ & $\Gamma\left({ }^{10} \operatorname{Be}\left(2_{1}^{+} ; \pi^{2}\right)\right)$ & $\Gamma\left({ }^{10} \mathrm{Be}\left(0_{2}^{+} ; \sigma^{2}\right)\right)$ & $\Gamma\left({ }^{10} \mathrm{Be}\left(1_{1}^{-} ; \pi \sigma\right)\right)$ & $\Gamma\left({ }^{10} \mathrm{Be}\left(2_{1}^{-} ; \pi \sigma\right)\right)$ & $\Gamma\left({ }^{6} \mathrm{He}+{ }^{8} \mathrm{Be}\right)$ \\
\hline $2^{+}$ & 22.93 & 0.4 & 0.2 & 99 & 0.1 & 0.1 & 29 \\
\hline $6^{+}$ & 26.45 & 0.2 & 0.4 & 42 & 0.2 & 0.6 & 17 \\
\hline $8^{+}$ & 29.39 & 0.2 & 0.8 & 17 & 2.9 & 5.6 & 13 \\
\hline \multicolumn{8}{|c|}{ (b) $\pi$-bond linear chain } \\
\hline
\end{tabular}

would expect that the decay of the $\sigma$-bond linear chain to the ${ }^{9} \mathrm{Be}$ ground state should be suppressed because of the $\pi$-bond nature of the ${ }^{9} \mathrm{Be}$ ground state. On the contrary, the decay to the first-excited state of ${ }^{9} \mathrm{Be}$ should be relatively enhanced because it has $\sigma$ bonding [24]. This decay pattern will also serve as more evidence of the $\sigma$-bond linear chain.

In conclusion, the $\pi$-bond linear-chain states decay to ${ }^{10} \mathrm{Be}\left(\pi^{2}\right)+\alpha$ and higher spin than the $6^{+}$states can decay to ${ }^{6} \mathrm{He}+\alpha+\alpha$. In the case of the $\sigma$-bond linear chain, they decay to ${ }^{10} \mathrm{Be}\left(\sigma^{2}\right)+\alpha$ and ${ }^{6} \mathrm{He}+\alpha+\alpha$ because all member states are above both threshold energies. This decay pattern constitutes important evidence to show the formation of two linear chains in ${ }^{14} \mathrm{C}$.

\section{SUMMARY}

In this paper, we focus on the linear-chain states of ${ }^{14} \mathrm{C}$ based on AMD calculations to establish the existence of the linear-chain configuration.

The linear-chain configurations generate two rotational bands. In the strongly deformed prolate region, two different linear-chain configurations with valence neutrons in a $\pi$ orbit and $\sigma$ orbit were obtained. The $\pi$-bond linear chain generates a rotational band around the $\alpha$ threshold energy. The energies and $\alpha$ decay widths of the $\pi$-bond linear chain are in reasonable agreement with the resonances observed by the $\alpha+{ }^{10} \mathrm{Be}$. Thus, the $\pi$-bond linear-chain formation in ${ }^{14} \mathrm{C}$ looks plausible.
On the other hand, the $\sigma$-bond linear-chain generates a rotational band around the ${ }^{6} \mathrm{He}$ threshold energy which is 7.5 MeV higher than the $\alpha$ threshold energy. Newly observed resonance states are close to energies of both the low-spin states of the $\sigma$-bond linear chain and the $6^{+}$state of the $\pi$-bond linear chain. To distinguish the $\pi$ - and $\sigma$-bond linear chain, we focus on their decay patterns. Reduced widths show that the $\pi$-bond linear-chain states decay into the ground band of ${ }^{10} \mathrm{Be}$, while the $\sigma$-bond linear-chain states decay into the excited band of ${ }^{10} \mathrm{Be}$. This difference is due to the molecular orbit of ${ }^{10} \mathrm{Be}$.

From ${ }^{6} \mathrm{He}$ reduced width, in addition, it is found that the $\sigma$-bond linear-chain states decay into not only the excited band of ${ }^{10} \mathrm{Be}$ but also ${ }^{6} \mathrm{He}+\alpha+\alpha$. Furthermore, the calculation predicts that the linear chain will also decay to the ${ }^{8,10} \mathrm{Be}\left(2^{+}\right)$ as well as to the ground state of ${ }^{8,10} \mathrm{Be}$. This characteristic decay patterns are, if observed, a strong signature of the $\pi$ and $\sigma$-bond linear-chain formations.

\section{ACKNOWLEDGMENTS}

The authors acknowledge the fruitful discussions with Dr. Suhara, Dr. Kanada-En'yo, Dr. Li, and Dr. Ye. One of the authors (M.K.) acknowledges the support by the Grants-inAid for Scientific Research on Innovative Areas from MEXT (Grant No. 2404:24105008) and JSPS KAKENHI Grant No. 16K05339. The other author (T.B.) acknowledges the support by JSPS KAKENHI Grant No. 16J04889.
[1] H. Morinaga, Phys. Rev. 101, 254 (1956).

[2] E. Uegaki, S. Okabe, Y. Abe, and H. Tanaka, Prog. Theor. Phys. 57, 1262 (1977); 62, 1621 (1979).

[3] M. Kamimura, Nucl. Phys. A 351, 456 (1981).

[4] P. Descouvemont and D. Baye, Phys. Rev. C 36, 54 (1987).

[5] Y. Kanada-En'yo, Phys. Rev. Lett. 81, 5291 (1998).

[6] A. Tohsaki, H. Horiuchi, P. Schuck, and G. Röpke, Phys. Rev. Lett. 87, 192501 (2001).

[7] Y. Funaki, A. Tohsaki, H. Horiuchi, P. Schuck, and G. Röpke, Phys. Rev. C 67, 051306 (2003).

[8] M. Chernykh, H. Feldmeier, T. Neff, P. von Neumann-Cosel, and A. Richter, Phys. Rev. Lett. 98, 032501 (2007).
[9] Y. Funaki, H. Horiuchi, and A. Tohsaki, Prog. Part. Nucl. Phys. 82, 78 (2015).

[10] Y. Funaki, Phys. Rev. C 94, 024344 (2016).

[11] N. Itagaki, S. Okabe, K. Ikeda, and I. Tanihata, Phys. Rev. C 64, 014301 (2001).

[12] W. von Oertzen and H. G. Bohlen, C. R. Phys. 4, 465 (2003).

[13] W. von Oertzen et al., Eur. Phys. J. A 21, 193 (2004).

[14] N. Itagaki, W. von Oertzen, and S. Okabe, Phys. Rev. C 74, 067304 (2006).

[15] T. Suhara and Y. Kanada-En'yo, Phys. Rev. C 82, 044301 (2010).

[16] J. Maruhn, N. Loebl, N. Itagaki, and M. Kimura, Nucl. Phys. A 833, 1 (2010). 
[17] N. Furutachi and M. Kimura, Phys. Rev. C 83, 021303(R) (2011).

[18] T. Suhara and Y. Kanada-En'yo, Phys. Rev. C 84, 024328 (2011)

[19] T. Baba, Y. Chiba, and M. Kimura, Phys. Rev. C 90, 064319 (2014).

[20] P. W. Zhao, N. Itagaki, and J. Meng, Phys. Rev. Lett. 115, 022501 (2015).

[21] M. Kimura, T. Suhara, and Y. Kanada-En'yo, Eur. Phys. J. A 52, 373 (2016).

[22] T. Baba and M. Kimura, Phys. Rev. C 94, 044303 (2016).

[23] M. Seya, M. Kohno, and S. Nagata, Prog. Theor. Phys. 65, 204 (1981).

[24] W. von Oertzen, Z. Phys. A 354, 37 (1996); 357, 355 (1997).

[25] Y. Kanada-En'yo, H. Horiuchi, and A. Doté, Phys. Rev. C 60, 064304 (1999).

[26] N. Itagaki and S. Okabe, Phys. Rev. C 61, 044306 (2000).

[27] N. Itagaki, S. Hirose, T. Otsuka, S. Okabe, and K. Ikeda, Phys. Rev. C 65, 044302 (2002).

[28] Y. Kanada-En'yo, M. Kimura, and H. Horiuchi, C. R. Phys. 4, 497 (2003).

[29] Y. Kanada-En'yo, M. Kimura, and A. Ono, Prog. Theor. Exp. Phys. 2012, 01A202 (2012).

[30] B. J. Greenhalgh, B. R. Fulton, D. L. Watson, N. M. Clarke, L. Donadille, M. Freer, P. J. Leask, W. N. Catford, K. L. Jones, and D. Mahboub, Phys. Rev. C 66, 027302 (2002).

[31] N. Soic et al., Phys. Rev. C 68, 014321 (2003).

[32] H. G. Bohlen et al., Phys. Rev. C 68, 054606 (2003).

[33] M. Milin et al., Nucl. Phys. A 730, 285 (2004).

[34] N. I. Ashwood et al., Phys. Rev. C 70, 064607 (2004).

[35] D. L. Price, M. Freer, N. I. Ashwood, N. M. Clarke, N. Curtis, L. Giot, V. Lima, P. Mc Ewan, B. Novatski, N. A. Orr, S. Sakuta, J. A. Scarpaci, D. Stepanov, and V. Ziman, Phys. Rev. C 75, 014305 (2007).

[36] M. Freer, J. D. Malcolm, N. L. Achouri, N. I. Ashwood, D. W. Bardayan, S. M. Brown, W. N. Catford, K. A. Chipps, J. Cizewski, N. Curtis, K. L. Jones, T. Munoz-Britton, S. D. Pain,
N. Soić, C. Wheldon, G. L. Wilson, and V. A. Ziman, Phys. Rev. C 90, 054324 (2014).

[37] A. Fritsch, S. Beceiro-Novo, D. Suzuki, W. Mittig, J. J. Kolata, T. Ahn, D. Bazin, F. D. Becchetti, B. Bucher, Z. Chajecki, X. Fang, M. Febbraro, A. M. Howard, Y. Kanada-En'yo, W. G. Lynch, A. J. Mitchell, M. Ojaruega, A. M. Rogers, A. Shore, T. Suhara, X. D. Tang, R. Torres-Isea, and H. Wang, Phys. Rev. C 93, 014321 (2016).

[38] D. Dell'Aquila, I. Lombardo, L. Acosta, R. Andolina, L. Auditore, G. Cardella, M. B. Chatterjiee, E. De Filippo, L. Francalanza, B. Gnoffo, G. Lanzalone, A. Pagano, E. V. Pagano, M. Papa, S. Pirrone, G. Politi, F. Porto, L. Quattrocchi, F. Rizzo, E. Rosato, P. Russotto, A. Trifirò, M. Trimarchi, G. Verde, and M. Vigilante, Phys. Rev. C 93, 024611 (2016).

[39] H. Yamaguchi et al., Phys. Lett. B 766, 11 (2017).

[40] Z. Y. Tian et al., Chin. Phys. C 40, 111001 (2016).

[41] J. Li, Y. L. Ye, Z. H. Li, C. J. Lin, Q. T. Li, Y. C. Ge, J. L. Lou, Z. Y. Tian, W. Jiang, Z. H. Yang, J. Feng, P. J. Li, J. Chen, Q. Liu, H. L. Zang, B. Yang, Y. Zhang, Z. Q. Chen, Y. Liu, X. H. Sun, J. Ma, H. M. Jia, X. X. Xu, L. Yang, N. R. Ma, and L. J. Sun, Phys. Rev. C 95, 021303(R) (2017).

[42] J. F. Berger, M. Girod, and D. Gogny, Comput. Phys. Commun. 63, 365 (1991).

[43] M. Kimura, Phys. Rev. C 69, 044319 (2004).

[44] M. Kimura, R. Yoshida, and M. Isaka, Prog. Theor. Phys. 127, 287 (2012).

[45] D. L. Hill and J. A. Wheeler, Phys. Rev. 89, 1102 (1953).

[46] Y. Kanada-En'yo, T. Suhara, and Y. Taniguchi, Prog. Theor. Exp. Phys. 2014, 073 D02 (2014).

[47] P. J. Haigh, N. I. Ashwood, T. Bloxham, N. Curtis, M. Freer, P. McEwan, D. Price, V. Ziman, H. G. Bohlen, T. Kokalova, C. Schulz, R. Torabi, W. von Oertzen, C. Wheldon, W. Catford, C. Harlin, R. Kalpakchieva, and T. N. Massey, Phys. Rev. C 78, 014319 (2008). 